Research Article

\title{
Local Buckling Analysis of T-Section Webs with Closed-Form Solutions
}

\author{
Cheng Liang, Yabo He, and Sumei Liu \\ School of Civil Engineering, Wuhan University, Wuhan 430072, China \\ Correspondence should be addressed to Yabo He; hyb5610@163.com
}

Received 15 October 2016; Accepted 20 November 2016

Academic Editor: Yakov Strelniker

Copyright (C) 2016 Cheng Liang et al. This is an open access article distributed under the Creative Commons Attribution License, which permits unrestricted use, distribution, and reproduction in any medium, provided the original work is properly cited.

This paper reports on approaches to estimate the critical buckling loads of thin-walled T-sections with closed-form solutions. We first develop a model using energy conservation approach under the assumption that there is no correlation between the restraint coefficient and buckling half-wavelength. Secondly, we propose a numerical approach to estimate the critical buckling conditions under the more realistic torsional stiffener constraint condition. A dimensionless parameter correlated with constraint conditions is introduced through finite element (FE) analysis and data fitting technique in the numerical approach. The critical buckling coefficient and loads can be expressed as explicit functions of the dimensionless parameter. The proposed numerical approach demonstrates higher accuracy than the approach under noncorrelation assumption. Due to the explicit expression of critical buckling loads, the numerical approach presented here can be easily used in the design, analysis, and precision manufacture of T-section webs.

\section{Introduction}

Thin-walled structures have been widely used in a large number of engineering applications such as civil infrastructure, high pressure vessel, aircraft composites, and wind turbines. Driven by those practical applications, considerable progress has been made in the design and analysis of composite elements such as beams and columns based on thin-walled structures [1-4]. Owing to their relatively low stiffness, local buckling generally occurs in thin-walled structures before reaching the strength failure of the materials, resulting in large deformation or even catastrophic failure of the overall system. Therefore, local buckling has been a critically important aspect in the design and precision manufacture of thinwalled structures $[5,6]$. Wattanutchariya and Paul proposed a model for the prediction of plate buckling during thermally enhanced microlamination process for reliability and stability of the manufacturing process [7]. Lee and Bang developed an approach for estimation of lateral buckling of a wind turbine tower with a thin circular wall through finite element (FE) analysis to ensure structure safety [8]. Kim et al. carried out a buckling analysis of filament-wound composite cylinder subject to deep water high hydrostatic pressure [9], and so forth.
Particularly, a great deal of research effort has also been carried out to study the buckling behaviour of thin-walled Tsections by considering the webs and flanges of thin-walled members as independent plates. In general, methods for determining the buckling loads of T-sections can be summarized into two categories [10]: (1) exact methods and (2) approximate methods. The former assume that the plates of the structures are simultaneously blended and the conjunctions of the plates satisfy continuity conditions, while the latter are also considered discrete plate analysis as they analyze the plates separately by taking the elastic restraints between adjacent plates into consideration. The discrete plate analysis approaches assume that the plate is simply supported at the loaded edges while being restrained by various conditions at the unloaded edge. For example, (1) the plate is elastically restrained at two unloaded edges (restrained-restrained (RR)) or (2) the plate is elastically restrained at one unloaded edge and free at the other unloaded edge (restrained-free $(\mathrm{RF})$ ), as shown in Figure 1.

When discrete plate analysis is used, it is necessary to determine the elastic restraint acting on the restrained plates. In 2001, Qiao et al. [5] obtained the critical buckling loads and half-wave numbers of axially loaded RF and RR plates 


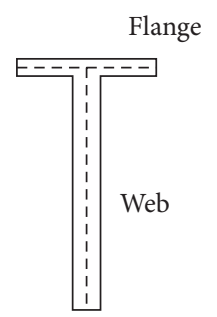

(a)

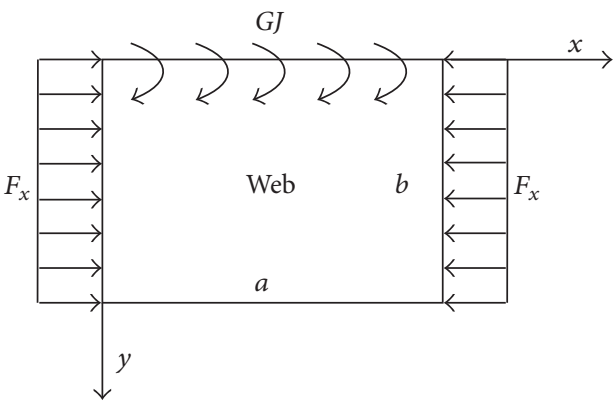

(b)

FIGURE 1: Schematics of restrained T-sections in this study: (a) cross-sectional view of T-sections; (b) uniaxially compressed T-section web restrained at one edge by torsional stiffeners and free at the other edge.

by solving two transcendental equations. They established a simplified expression for the critical buckling loads related to elastic restraint coefficients by using a fitting technique. Based on the derivation process conducted by Bleich et al. under isotropic conditions [11], Qiao presented conservative and simplified expressions for the restraint coefficients of rotating springs for I- and box-sections under anisotropic conditions by regarding the restraints as rotational spring constraints. Moreover, Qiao and Shan [6] presented an expression for the rotational restraint stiffness $k$ for various thin-walled elements through the design and analysis of the local buckling of fibre-reinforced plastic composite. By regarding the webs and flanges of T-section members as a whole, they performed a finite element (FE) analysis to calculate the rotational restraint stiffness. Meanwhile, by using the fitting technique, they derived expressions for the rotational restraint stiffness of the webs and flanges of the T-section members, respectively. However, this method cannot be generally used since the rotational stiffness of any new section needs to be fitted again. Moreover, all of the analysis proposed by Qiao and Beich is based on the assumption that the restraints provided by the Tsection flange to the web section are equivalent to rotational springs; that is, the restraining bending moment of the flange to the web of a unit length is

$$
M_{x}=k \frac{\partial w}{\partial y}=k \theta,
$$

where $\theta$ is the angle between the flange and the web; $k$ is the rotational restraint stiffness.

However, the rotational spring restraint assumption is not always valid; Kollar [10, 12-14] divided the elastic restraints into two kinds based on analysis of the configurations of various sections: (1) when both of the two edges of the restrained plates are restrained, the restraints are equivalent to a rotating spring [11] and (2) when one edge of the restrained plates is free, the restraints are equivalent to torsional stiffener [15]. The former occurs when the webs and flanges of box-sections and the webs of I-, C-, and Z-sections are used as restrained plates, as shown in Figure 2(a). While, when the flanges of I-, C-, and Z-sections act as the restrained plates, the latter appears, as shown in Figure 2(b). In the case of Figure 2(b), the bending moments at the edges of the restrained web are equal to the distributed torque imposed by the flange plates and are related to the rate of change of the angle $\theta^{\prime}$. As shown in Figure 3, the restraining bending moment at the intersection of the flange and the web per unit length is

$$
M_{x}=\left(G J \theta^{\prime}\right)^{\prime}=G J \frac{\partial^{3} w}{\partial x^{2} \partial y},
$$

where $G J$ represents the torsional stiffness of the flange-web restraint.

In addition, Kollar presented an expression for the torsional restraining stiffness and a simple explicit expression for the critical buckling loads of RF [10] and RR [16] plates. However, this expression is not rigorous in theory and cannot be used to obtain closed-form solutions.

In this work, we propose explicit solutions for critical buckling load of the T-section webs based on the torsional restraint model through Rayleigh-Ritz method and finite element (FE) analysis. Firstly, a closed-form expression for the critical buckling load of T-section webs is established using energy conservation approach with the assumption of noncorrelation of the restrain coefficient and buckling halfwavelength. Then, based on the FE analysis and the fitting technique, an explicit expression for the buckling half-wavelength is provided to calculate the restraint coefficients of torsional stiffeners, which is more realistic than the noncorrelation assumption. By introducing a dimensionless parameter $\beta$ in the numerical approach, we present an explicit expression for the critical buckling load which can be directly applied to the design of the thin-walled of T-section structures.

\section{Variational Formula for the Local Buckling of Elastically Restrained Plates}

Based on the discrete plate analysis proposed in the above section, both the web and flange of a T-section component can be simulated as RF plate units free at one unloaded edge and elastically restrained at the other unloaded edge, as shown in Figure 1. Moreover, the web and the flange can be regarded as being restrained by torsional stiffeners. Here, the variational formula is adopted to analyze the local buckling of an isotropic plate with the boundary conditions presented in Figure 1 using the Rayleigh-Ritz method. Measuring $a$ in 


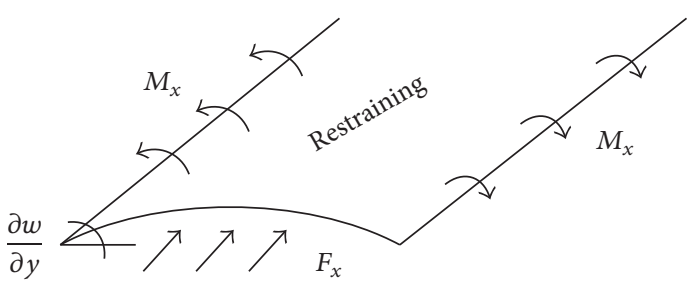

(a)

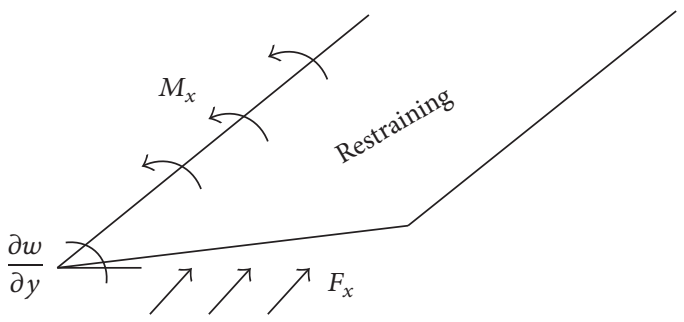

(b)

FIgURE 2: Restrained plates. (a) A restrained plate whose two edges are adjacent to the plate; (b) restrained plate with a free edge.

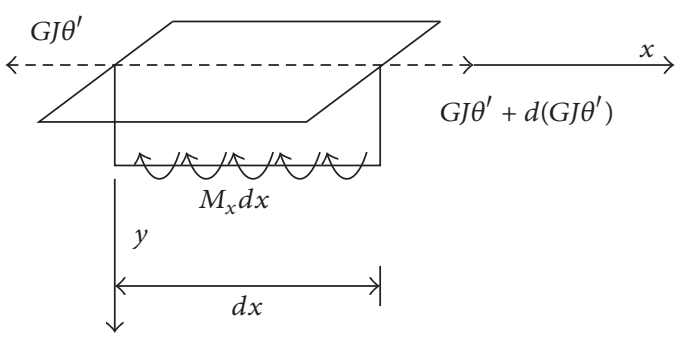

FIGURE 3: Elastic restraints of the flange on the web of the T-section: torque balance over a small part of the flange.

length and $b$ in width, the rectangular plate is uniformly loaded in the $x$-direction. The boundary parallel to the $y$-axis is simply supported. The $y=b$ edge is a free boundary, while the $y=0$ edge is the boundary restrained by torsional stiffeners with torsional stiffness GJ. In this plate system, the total potential energy $\Pi$ is composed of three parts: (1) the elastic strain energy $U_{e}$ stored in the plate, (2) the restraint strain energy $U_{r}$ preserved in the torsional stiffener constraint, and (3) the work $V$ done by the externally applied force. These three parts can be calculated by using the appropriate function $w$ as the buckling displacement out of the plane of the plate.

The elastic strain energy $U_{e}$, restraint strain energy $U_{r}$, and the work $V$ done by the external force for orthogonal isotropic plates can be expressed as follows:

$$
\begin{aligned}
U_{e} & =\frac{1}{2} \iint D\left\{\left(\nabla^{2} w\right)^{2}-2(1-\mu)\right. \\
\cdot & {\left.\left[\frac{\partial^{2} w}{\partial x^{2}} \frac{\partial^{2} w}{\partial y^{2}}-\left(\frac{\partial^{2} w}{\partial x \partial y}\right)^{2}\right]\right\} d x d y } \\
& =\frac{D}{2} \iint\left[w_{, x x}^{2}+w_{, y y}^{2}+2 \mu w_{, x x} w_{, y y}+2(1-\mu)\right. \\
& \left.\cdot w_{, x y}^{2}\right] d x d y, \\
U_{r} & =-\frac{1}{2} \int\left[G J\left(\left.\frac{\partial^{3} w}{\partial x^{2} y}\right|_{y=0}\right)\left(\left.\frac{\partial w}{\partial y}\right|_{y=0}\right)\right] d x, \\
V & =\frac{1}{2} \iint\left[F_{x}\left(\frac{\partial w}{\partial x}\right)^{2}\right] d x d y,
\end{aligned}
$$

where $D=E t^{3} / 12\left(1-\mu^{2}\right), E, t$, and $\mu$ are the flexural rigidity, elastic modulus, thickness, and Poisson's ratio of the plate, respectively; $G J$ represents the torsional stiffness of the torsional stiffener per unit length at $y=0 ; F_{x}$ is the axial force per unit length of the loaded edge of the plate, while $w$ denotes the buckling shape function of the plate.

Considering small deformations and only the linearelastic behaviour of the plate, the first-order variational form is presented as follows:

$$
\begin{gathered}
\delta U_{e}=D \iint\left[w_{, x x} \delta w_{, x x}+w_{, y y} \delta w_{, y y}+\mu\left(w_{, y y} \delta w_{, x x}\right.\right. \\
\left.\left.+w_{, x x} \delta w_{, y y}\right)+2(1-\mu) w_{, x y} \delta w_{, x y}\right] d x d y, \\
\delta U_{r}=-\frac{1}{2} \int\left\{G J \left[\left(\left.\frac{\partial^{3} w}{\partial x^{2} y}\right|_{y=0}\right) \delta\left(\left.\frac{\partial w}{\partial y}\right|_{y=0}\right)\right.\right. \\
\left.\left.\quad+\left(\left.\frac{\partial w}{\partial y}\right|_{y=0}\right) \delta\left(\left.\frac{\partial^{3} w}{\partial x^{2} y}\right|_{y=0}\right)\right]\right\} d x, \\
\delta V=\iint\left(F_{x} w_{, x} \delta w_{, x}\right) d x d y .
\end{gathered}
$$

For a plate system in an equilibrium state, the first-order variational form of its total potential energy equals zero; that is,

$$
\delta \Pi=\delta U_{1}+\delta U_{2}-\delta V=0 .
$$

By substituting the buckling shape function $w$ satisfying certain boundary conditions into (7), we can establish a standard buckling eigenvalue problem which can be solved using the Rayleigh-Ritz method.

\section{Explicit Solutions of the Local Buckling of Elastically Restrained Plates}

When solving the buckling problem using the Rayleigh-Ritz method, it is crucial to select an appropriate shape function $w$ [17]. This function is expected to satisfy certain boundary restraints including force boundary restraints and displacement boundary restraints so as to ensure accuracy. Moreover, the structural form of the function is chosen to be as simple as possible for the convenience of obtaining corresponding explicit formula. Based on the recommendations of selecting 
shape functions in relevant studies $[18,19]$, the shape function shown in (8) is used to simulate the buckling deformation of plate units

$$
w=\left[\frac{y}{b}+\psi_{1}\left(\frac{y}{b}\right)^{2}+\psi_{2}\left(\frac{y}{b}\right)^{3}+\psi_{3}\left(\frac{y}{b}\right)^{4}\right] \sin \frac{\pi x}{a_{0}},
$$

where $\psi_{1}-\psi_{3}$ are coefficients determined by the boundary constraint conditions; $a_{0}=a / i$ is the buckling halfwavelength and $i$ is the buckling half-wave number $(i=$ $1,2, \ldots)$.

The shape function used here applies strictly defined force boundary constraints at the unloaded edges. The specific expressions are presented in $(9 \mathrm{a})$ to $(9 \mathrm{~d})$

$$
w(x, 0)=0 .
$$

The torsional stiffener restraint is

$$
\begin{aligned}
M_{x}(x, 0) & =-D\left(\left.\frac{\partial^{2} w}{\partial y^{2}}\right|_{y=0}\right)=G J\left(\left.\frac{\partial^{3} w}{\partial x^{2} \partial y}\right|_{y=0}\right), \\
M_{x}(x, b) & =\left.\left(\frac{\partial^{2} w}{\partial y^{2}}+\mu \frac{\partial^{2} w}{\partial x^{2}}\right)\right|_{y=b}=0, \\
V_{y} & =\left.\left(\frac{\partial^{3} w}{\partial y^{3}}+(2-\mu) \frac{\partial^{3} w}{\partial x^{2} y}\right)\right|_{y=b}=0 .
\end{aligned}
$$

By substituting (8) into (9a)-(9d), specific expressions for the coefficients $\psi_{1}-\psi_{3}$ can be obtained, as shown in (10a) to (10c)

$$
\begin{aligned}
& \psi_{1}=\frac{G J \pi^{2} b}{2 D a_{0}^{2}}, \\
& \psi_{2}=-\frac{\left(48 a_{0}^{4} \psi_{1}+3 \pi^{4} b^{4}+2 \pi^{4} b^{4} \psi_{1}-12 \pi^{2} a_{0}^{2} b^{2}-3 \pi^{4} b^{4} s^{2}+36 \pi^{2} a_{0}^{2} b^{2} s-2 \pi^{4} b^{4} s^{2} \psi_{1}-8 \pi^{2} a_{0}^{2} b^{2} \psi_{1}+40 \pi^{2} a_{0}^{2} b^{2} s \psi_{1}\right)}{\left(72 a_{0}^{4}+30 \pi^{2} a_{0}^{2} b^{2} s-6 \pi^{2} a_{0}^{2} b^{2}-\pi^{4} b^{4} s^{2}+\pi^{4} b^{4}\right)} \\
& \psi_{3}=\frac{\left(12 a_{0}^{4} \psi_{1}+2 \pi^{4} b^{4}+\pi^{4} b^{4} \psi_{1}-2 \pi^{4} b^{4} s^{2}+12 \pi^{2} a_{0}^{2} b^{2} s-\pi^{4} b^{4} s^{2} \psi_{1}+12 \pi^{2} a_{0}^{2} b^{2} s \psi_{1}\right)}{\left(72 a_{0}^{4}+30 \pi^{2} a_{0}^{2} b^{2} s-6 \pi^{2} a_{0}^{2} b^{2}-\pi^{4} b^{4} s^{2}+\pi^{4} b^{4}\right)},
\end{aligned}
$$

where $s=1-\mu$ and $\psi_{1}$ is termed as the restraint coefficient and $\psi_{1}=0$ indicates that the plate is simply supported; when $\psi_{1}=\infty$, the plate is clamped.

3.1. Plates of Finite Length. By substituting (8) into (7), the corresponding analytical solutions for buckling loads and buckling coefficient after solving the standard eigenvalue problem can be derived. The specific expressions are shown as follows:

$$
\begin{aligned}
\left(F_{x}\right)_{\mathrm{cr}} & =K_{\mathrm{cr}} \frac{\pi^{2} D}{b^{2}} \\
K_{\mathrm{cr}} & =\frac{b^{2}}{a_{0}^{2}}+\frac{a_{0}^{2}}{b^{2}}\left(\frac{2 \eta_{2}+\psi_{1}}{2 \pi^{4} \eta_{1}}\right)+\frac{(1-\mu) \eta_{3}+\eta_{4}}{\pi^{2} \eta_{1}}
\end{aligned}
$$

where $\left(F_{x}\right)_{\text {cr }}$ and $(K)_{\text {cr }}$ are the critical buckling load and buckling coefficient, respectively. For each buckling halfwave number $i$, there is a corresponding critical buckling load $\left(F_{x}\right)_{\mathrm{cr}}$. We are interested in finding the minimum value of $\left(F_{x}\right)_{\mathrm{cr}}$. In (12) $\eta_{1}-\eta_{4}$ are all functions of $\psi_{1}$

$$
\begin{aligned}
& \eta_{1}=\frac{\left(252 \psi_{1}^{2}+180 \psi_{2}^{2}+140 \psi_{3}^{2}+420 \psi_{1} \psi_{2}+360 \psi_{1} \psi_{3}+315 \psi_{2} \psi_{3}+630 \psi_{1}+504 \psi_{2}+420 \psi_{3}+420\right)}{5040}, \\
& \eta_{2}=\frac{\left(5 \psi_{1}^{2}+15 \psi_{2}^{2}+36 \psi_{3}^{2}+15 \psi_{1} \psi_{2}+20 \psi_{1} \psi_{3}+45 \psi_{2} \psi_{3}\right)}{5}, \\
& \eta_{3}=\psi_{1}^{2}+1.5 \psi_{2}^{2}+2 \psi_{3}^{2}+2.5 \psi_{1} \psi_{2}+3 \psi_{1} \psi_{3}+3.5 \psi_{2} \psi_{3}+1.5 \psi_{1}+2 \psi_{2}+2.5 \psi_{3}+0.5 \\
& \eta_{4}=-\frac{\left(70 \psi_{1}^{2}+126 \psi_{2}^{2}+180 \psi_{3}^{2}+210 \psi_{1} \psi_{2}+294 \psi_{1} \psi_{3}+315 \psi_{2} \psi_{3}+105 \psi_{1}+210 \psi_{2}+315 \psi_{3}\right)}{210} .
\end{aligned}
$$

3.2. Long Plates. In this section, long plates with $a \gg b$ are investigated to obtain the minimal critical buckling load as a function of buckling half-wavelength $a_{0}$. To get the minimal value of $\left(F_{x}\right)_{\mathrm{cr}}$, it is necessary to make $d\left(F_{x}\right)_{\mathrm{cr}} / d a_{0}=0$. An explicit expression for the critical aspect ratio may be found if the restraint coefficients of the rotating springs of RF plates are not related to $a_{0}$, as pointed out in the literature [6]. However, when using torsional stiffeners to simulate the restraints 
of T-sections, the restraint coefficient $\psi_{1}$ of torsional stiffeners of RF plates (as presented in (10a)) is relevant to $a_{0}$. This condition results in a transcendental equation, which can be only solved numerically. In the following sections, we present two approaches to derive explicit solutions for the critical buckling load.

\subsubsection{Energy Conservation Approach under Noncorrelation} Assumption. To obtain an explicit solution, we assume there is no correlation between $\psi_{1}$ and $a_{0}$. With the shape function shown in (8) and energy conservation condition shown in (7), for a long plate with one edge restrained by rotating springs and one free edge, the critical buckling load and coefficient can be derived as

$$
\begin{aligned}
\left(F_{x}\right)_{\mathrm{cr}} & =K_{\mathrm{cr}} \frac{\pi^{2} D}{b^{2}}, \\
K_{\mathrm{cr}} & =2 \sqrt{\frac{2 \eta_{2}+\psi_{1}}{2 \pi^{4} \eta_{1}}}+\frac{(1-\mu) \eta_{3}+\eta_{4}}{\pi^{2} \eta_{1}} .
\end{aligned}
$$

Specifically, $\psi_{1}=0$ indicates that the plate is simply supported; the buckling coefficient is $K_{\mathrm{sf}}$; when $\psi_{1}=\infty$, the plate is clamped and the buckling coefficient is $K_{\mathrm{cf}}$

$$
\begin{aligned}
& K_{\mathrm{sf}}=0.608(1-\mu), \\
& K_{\mathrm{cf}}=1.53 \sqrt{1-\mu} .
\end{aligned}
$$

\subsubsection{Numerical Approach under Torsional Stiffener Restraint} Assumption. As presented earlier, the critical buckling load of those plates restrained by torsional stiffeners is related to the restraint coefficient $\psi_{1}$. Meanwhile, the restraint coefficient $\psi_{1}$ of a torsional stiffener is related to the buckling halfwavelength $a_{0}$. As analytical solutions cannot be obtained for $a_{0}$, a simple explicit expression for $a_{0}$ based on the FE analysis and the fitting technique will be presented below.

By using FEA software ANSYS, the model of the whole structure of the T-section shown in Figure 4 is established to analyze the elastic buckling of the web under uniform compressive stress. To highlight the torsional stiffener restraints on the flange to the web, degree of freedom (DOF) coupling is adopted in the modelling process. The flange and the web are separately modelled. Afterwards, rotational freedoms 4 and 5 of the nodes on the intersection of the web and the flange are coupled with the displacement DOF 3 outside the web (other DOFs remain independent). The model aims to prevent the uniform compressive stress imposed on the web from being transferred to the flange, thus avoiding buckling of the flange. Meanwhile, it is necessary to restrain DOFs 1 and 2 of nodes at both ends of the flange in order to prevent the flange from rigid translational movement. In the ANSYS model, loads are applied to the nodes on the loading edges by means of nodal loads. The value of the nodal load is calculated based on the tributary area of nodes: the load on tributary area of intermediate nodes is the sum of the half-areas of the two units adjacent to this node, while that on end nodes is equal to half of its unit value.

By analyzing a large number of models with varying $b_{f}$, $t_{f}, b_{w}$, and $t_{w}\left(b_{w}\right.$ and $t_{w}$ refer to the width and thickness of

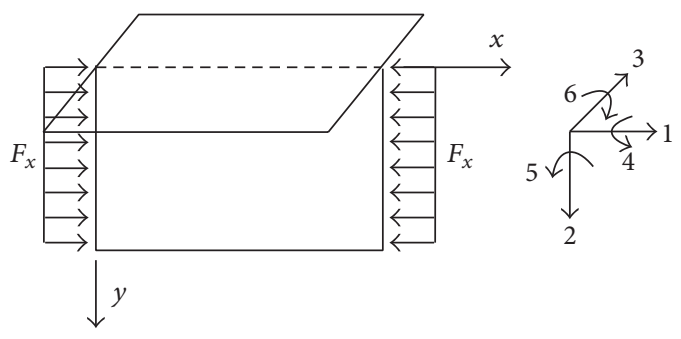

FIgURE 4: The web being uniaxially compressed by uniform loads (considering flange restraint).

the web; $b_{f}$ and $t_{f}$ refer to the width and thickness of the flange) and using data fitting technique, the buckling halfwavelength $a_{0}$ can be expressed as follows:

$$
a_{0}=\frac{1.675}{1-0.39 \beta^{-0.655}} b
$$

where the introduced variable $\beta=b_{f} t_{f}^{3} / b_{w} t_{w}^{3}$ refers to the ratio of the torsional stiffness of the flange to the bending stiffness of the web. When $\beta=0.2375$, the constrained edge of the web is equivalent to a simply supported edge with a buckling half-wavelength $a_{0}$ being the length $a$ of the plate. It also indicates that the flange of the T-section cannot restrain the web when $b_{f} t_{f}^{3}<0.2375 b_{w} t_{w}^{3}$. In addition, when $\beta=\infty$, the constrained edge of the web is equivalent to the simply supported edge with the buckling half-wavelength being $a_{0}=$ $1.675 b$.

By substituting (10a)-(10c), (13a)-(13d), and (17) into (15), the critical buckling load of a long plate can be calculated under the noncorrelation assumption. Take a T-section shown in Figure 4, for example, where the parameters of the T-section are follows: $b_{w}=6 \mathrm{~cm}$ and $t_{w}=t_{f}=0.25 \mathrm{~cm} ; b_{f}$ is a variable, which means that $\beta$ is also a variable. Figure 5 shows that, compared with the critical buckling load of the T-section calculated by the FE analysis, the buckling loads of the RF plate computed by (15) under the noncorrelation assumption present a maximum error of $3.52 \%$.

Substituting (17) into (10a) gives

$$
\begin{aligned}
\psi_{1} & =\frac{G J \pi^{2} b}{2 D a_{0}^{2}}=\frac{s \pi^{2} b_{f} t_{f}^{3}}{\left(1.675 /\left(1-0.39 \beta^{-0.655}\right)\right)^{2} b_{w} t_{w}^{3}} \\
& =\frac{s \pi^{2} \beta}{\left(1.675 /\left(1-0.39 \beta^{-0.655}\right)\right)^{2}} .
\end{aligned}
$$

An interesting point arising from (18) is that the restraint coefficient $\psi_{1}$ can be directly correlated with $\beta$. This indicates that the critical buckling load and coefficient can also be derived as explicit functions of $\beta$. On this basis, an explicit expression, simpler than that in (15), was obtained using the fitting technique

$$
K_{\mathrm{cr}}=K_{\mathrm{sf}}+\left(K_{\mathrm{cf}}-K_{\mathrm{sf}}\right) * 0.5^{1 / \beta},
$$

where $K_{\mathrm{sf}}$ and $K_{\mathrm{cf}}$ are given by (16a) and (16b). 


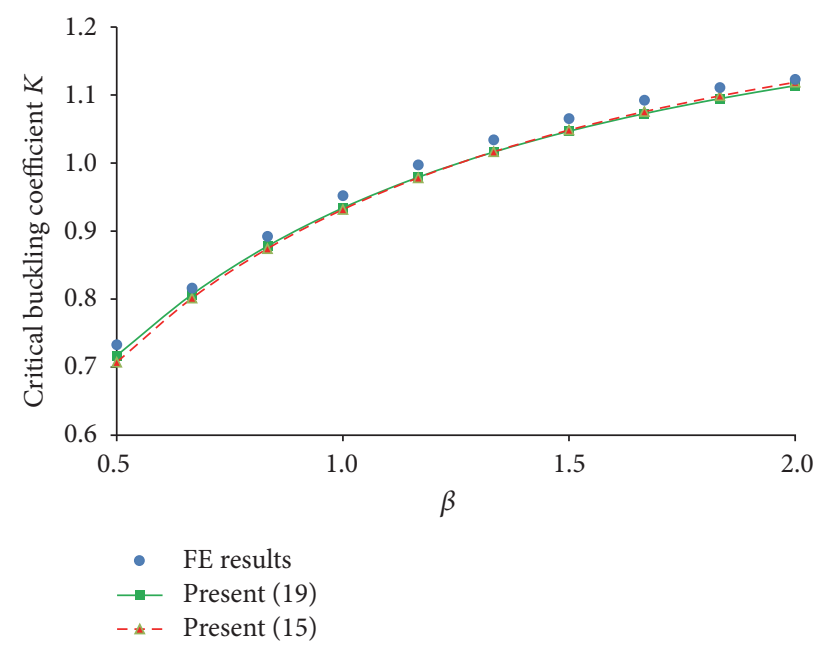

Figure 5: Comparison of the critical buckling coefficient of the RF plate calculated using three methods.

As shown in Figure 5, compared with the critical buckling load calculated by the FE approach, the critical buckling load computed by (19) is more conservative with a maximum error of only $2.18 \%$. The closed-form solution of the critical buckling load coefficient presented in (19) demonstrates higher accuracy than (15) under the noncorrelation assumption, as well as simplicity for practical implementation in engineering applications.

\section{Conclusion}

We present closed-form solutions for the critical buckling loads of thin-walled T-section structures. An explicit expression of the critical buckling loads is derived based on energy conservation and constraint coefficient-buckling halfwavelength noncorrelation assumption. Secondly, we develop a numerical approach by taking account of the correlation of the constraint coefficient and buckling half-wavelength. Through FE analysis and data fitting, the buckling halfwavelength $a_{0}$ can be correlated with the geometric parameters of the T-section via a variable $\beta$. $\beta$ is not merely a data fitting parameter but also directly corresponds to various constraint conditions. It is found that the restraint coefficient $\psi_{1}$ can be expressed as a closed-form solution of $\beta$ under energy conservation approach. Therefore, the critical buckling load and coefficient can also be derived as explicit functions of $\beta$.

The explicit expression of critical buckling coefficient [see (19)] derived by the numerical approach provides benefits of not only convenient applications in engineering practice, but also higher accuracy than the explicit expression obtained under the noncorrelation assumption [see (15)]. We expect the closed-form solutions proposed herein to be valuable for design and precision manufacture of T-section structures.

\section{Competing Interests}

The authors declare that there is no conflict of interests regarding the publication of this paper.

\section{References}

[1] L. C. Bank and J. Yin, "Buckling of orthotropic plates with free and rotationally restrained unloaded edges," Thin-Walled Structures, vol. 24, no. 1, pp. 83-96, 1996.

[2] J. F. Davalos, H. A. Salim, P. Qiao, R. Lopez-Anido, and E. J. Barbero, "Analysis and design of pultruded FRP shapes under bending," Composites Part B: Engineering, vol. 27, no. 3-4, pp. 295-305, 1996.

[3] J. F. Davalos and P. Qiao, "A computational approach for analysis and optimal design of FRP beams," Computers and Structures, vol. 70, no. 2, pp. 169-183, 1999.

[4] P. Qiao, J. F. Davalos, and B. Brown, "A systematic analysis and design approach for single-span FRP deck/stringer bridges," Composites Part B: Engineering, vol. 31, no. 6-7, pp. 593-609, 2000.

[5] P. Qiao, J. F. Davalos, and J. Wang, "Local buckling of composite FRP shapes by discrete plate analysis," Journal of Structural Engineering, vol. 127, no. 3, pp. 245-255, 2001.

[6] P. Qiao and L. Shan, "Explicit local buckling analysis and design of fiber-reinforced plastic composite structural shapes," Composite Structures, vol. 70, no. 4, pp. 468-483, 2005.

[7] W. Wattanutchariya and B. K. Paul, "Bonding fixture tolerances for high-volume metal microlamination based on fin buckling and laminae misalignment behavior," Precision Engineering, vol. 28, no. 2, pp. 117-128, 2004.

[8] K.-S. Lee and H.-J. Bang, "A study on the prediction of lateral buckling load for wind turbine tower structures," International Journal of Precision Engineering \& Manufacturing, vol. 13, no. 10, pp. 1829-1836, 2012.

[9] M.-H. Kim, J.-R. Cho, W.-B. Bae et al., "Buckling analysis of filament-wound thick composite cylinder under hydrostatic pressure," International Journal of Precision Engineering \& Manufacturing, vol. 11, no. 6, pp. 909-913, 2010.

[10] L. P. Kollár, "Local buckling of fiber reinforced plastic composite structural members with open and closed cross sections," Journal of Structural Engineering, vol. 129, no. 11, pp. 1503-1513, 2003.

[11] F. Bleich, L. B. Ramsey, and H. H. Bleich, Buckling Strength of Metal Structures, McGraw-Hill, New York, NY, USA, 1952.

[12] G. Tarján, Á. Sapkás, and L. P. Kollár, "Local web buckling of composite (FRP) beams," Journal of Reinforced Plastics \& Composites, vol. 29, no. 10, pp. 1451-1462, 2010.

[13] G. Tarján, Á. Sapkás, and L. P. Kollár, "Stability analysis of long composite plates with restrained edges subjected to shear and linearly varying loads," Journal of Reinforced Plastics and Composites, vol. 29, no. 9, pp. 1386-1398, 2010.

[14] L. P. Kollár, "Buckling of unidirectionally loaded composite plates with one free and one rotationally restrained unloaded edge," Journal of Structural Engineering, vol. 128, no. 9, pp. 1202 1211, 2002.

[15] S. P. Timoshenko, Theory of Elastic Stability, McGraw-Hill, New York, NY, USA, 1961.

[16] L. P. Kollár, "Discussion of "local buckling of composite FRP shapes by discrete plate analysis" by Pizhong Qiao, Julio F. Davalos, and Jialai Wang," Journal of Structural Engineering, vol. 128, no. 8, pp. 1091-1093, 2002.

[17] E. J. Barbero and I. G. Raftoyiannis, "Local buckling of FRP beams and columns," Journal of Materials in Civil Engineering, vol. 5, no. 3, pp. 339-355, 1993. 
[18] P. Qiao and G. Zou, "Local buckling of elastically restrained fiber-reinforced plastic plates and its application to box sections," Journal of Engineering Mechanics, vol. 128, no. 12, pp. 1324-1330, 2002.

[19] P. Qiao and G. Zou, "Local buckling of composite fiber-reinforced plastic wide-flange sections," Journal of Structural Engineering, vol. 129, no. 1, pp. 125-129, 2003. 


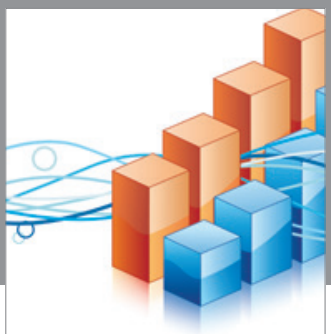

Advances in

Operations Research

vatem alat4

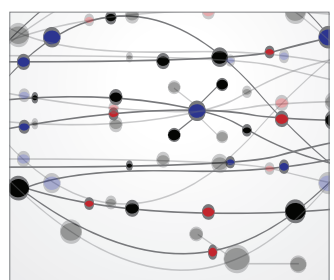

\section{The Scientific} World Journal
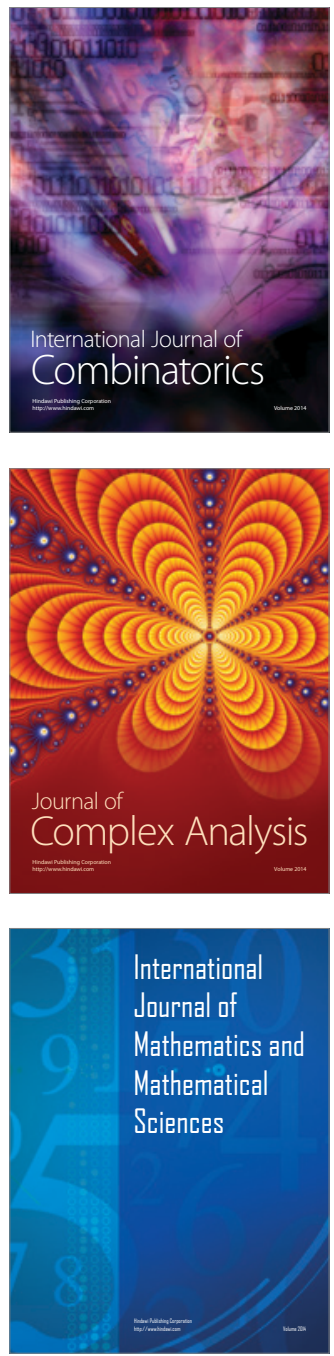
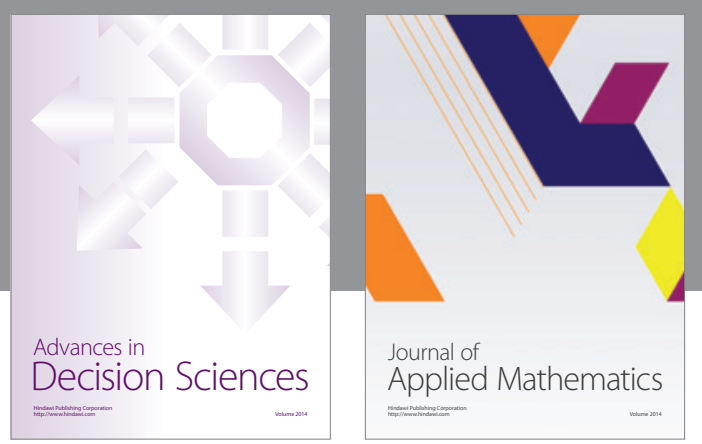

Algebra

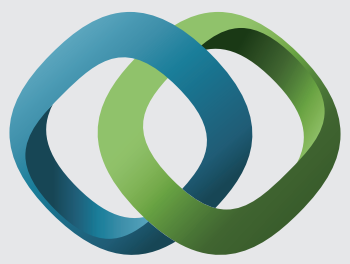

\section{Hindawi}

Submit your manuscripts at

http://www.hindawi.com
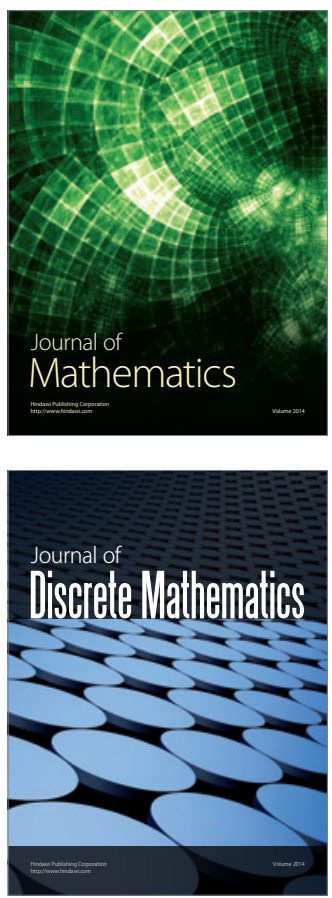

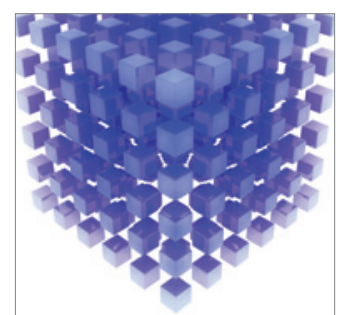

Mathematical Problems in Engineering
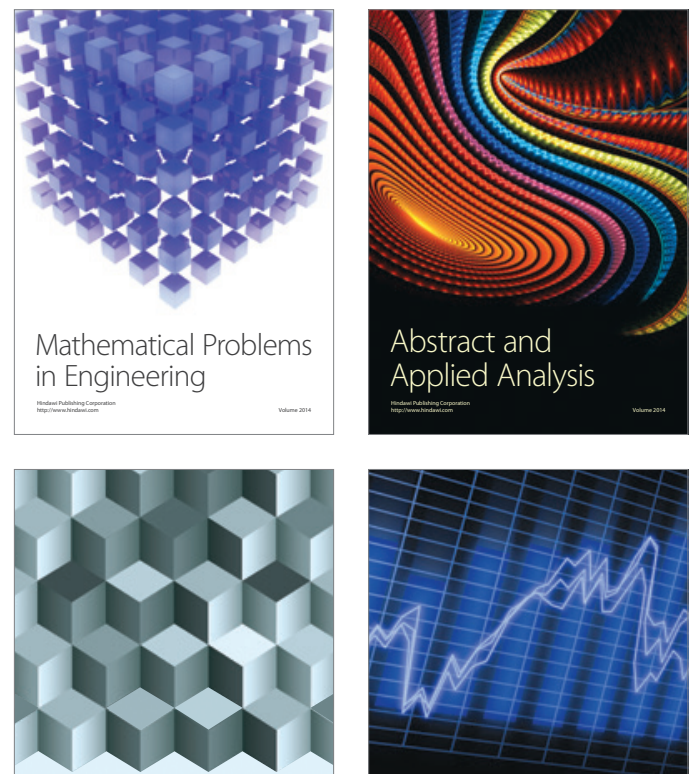

Journal of

Function Spaces

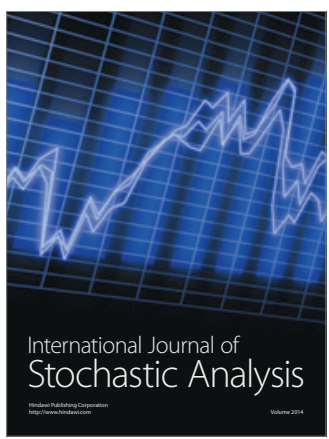

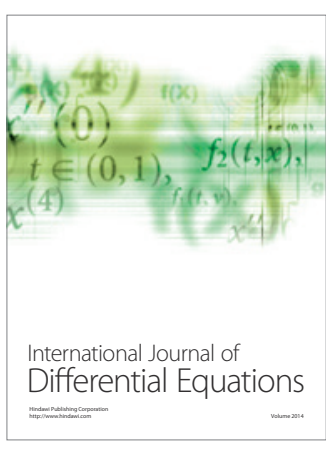
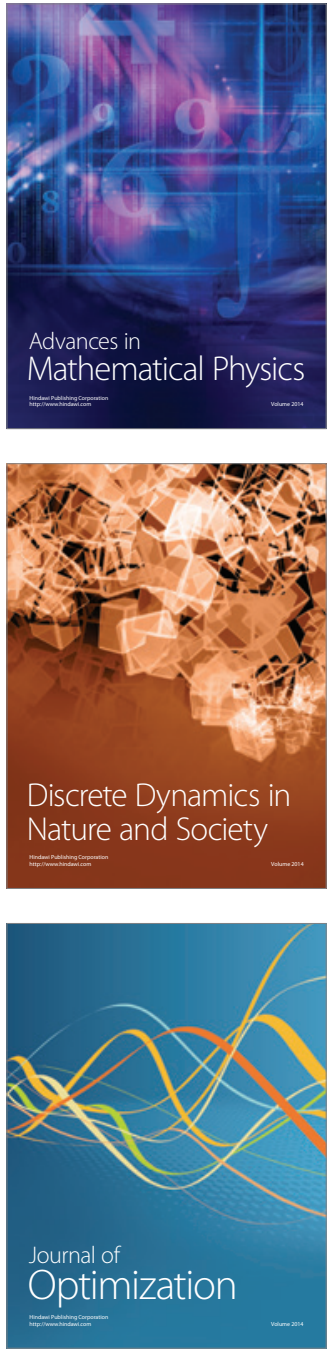